\title{
Berberine inhibits the LPS-induced proliferation and inflammatory response of stromal cells of adenomyosis tissues mediated by the LPS/TLR4 signaling pathway
}

\author{
LI LIU, LI CHEN, CAIXIA JIANG, JING GUO, YAN XIE, LE KANG and ZHONGPING CHENG \\ Department of Obstetrics and Gynecology, Yangpu Hospital, \\ Tongji University School of Medicine, Shanghai 200090, P.R. China
}

Received March 24, 2017; Accepted August 15, 2017

DOI: $10.3892 /$ etm.2017.5316

\begin{abstract}
A previous study by our group has demonstrated that lipopolysaccharide (LPS) induces adenomyosis through stimulating inflammatory cell proliferation and invasive growth of stromal cells via Toll-like receptor 4 (TLR4) signaling. The present study aimed to investigate the effects of berberine (BBR) on LPS-induced ectopic endometrial stromal cells (EESCs) isolated from patients with adenomyosis. The viability of EESCs treated with LPS or LPS plus BBR was detected by a cell counting kit- 8 assay, and the cell cycle distribution and apoptosis were evaluated by flow cytometry. The effect of BBR on the expression of key molecules of inflammatory proliferation and invasive growth of LPS-induced EESCs was also evaluated. BBR significantly inhibited the LPS-induced proliferation of EESCs in a dose- and time-dependent manner. BBR induced cell cycle arrest in G0/G1 phase and enhanced apoptosis of LPS-induced EESCs. Furthermore, BBR inhibited the expression of interleukin (IL)-6, IL-8, transforming growth factor- $\beta$, epithelial growth factor, vascular endothelial growth factor and matrix metalloproteinase 2 in LPS-induced EESCs. To the best of our knowledge, the present study was the first to demonstrate that BBR has a protective effect on ameliorating the LPS-induced progression of adenomyosis. This result may provide a novel therapeutic strategy for the clinical treatment of the disease.
\end{abstract}

\section{Introduction}

Adenomyosis is a common chronic gynecological disorder characterized by penetration of ectopic endometrium into the

Correspondence to: Dr Zhongping Cheng, Department of Obstetrics and Gynecology, Yangpu Hospital, Tongji University School of Medicine, 450 Tengyue Road, Shanghai 200090, P.R. China

E-mail: mdcheng18@263.net

Keywords: berberine,adenomyosis,stromalcells,lipopolysaccharide, proliferation surrounding myometrium. As a special form of endometriosis, the typical clinical features of adenomyosis include menorrhagia, dysmenorrhea, metrorrhagia and infertility (1-3). The medications most commonly used to treatments for adenomyosis are hormonal, including the levonorgestrel intrauterine system and gonatropin-releasing hormone agonists. In consideration of the inflammatory pathology of adenomyosis, other non-hormonal treatments, including non-steroidal anti-inflammatory drugs, are also effective for dysmenorrhea in adenomyosis. However, the exact pathogenesis of adenomyosis has remained elusive. Emerging evidence suggested that this disease may be linked to the expression of inflammatory mediators and the induction of an immune response (4-6). A previous study by our group has confirmed that lipopolysaccharide (LPS) stimulates inflammatory cell proliferation and invasive growth of stromal cells in adenomyosis by activation of the Toll-like receptor (TLR4)/myeloid differentiation primary response gene 88 /nuclear factor (NF)- $\kappa$ B signaling pathway, indicating that LPS/TLR4 signaling is implicated in the pathogenesis of adenomyosis (7). This provided a novel therapeutic strategy for adenomyosis via targeting TLR4 signaling.

Berberine (BBR), extracted from rhizoma coptis (Huanglian in Chinese) and other Chinese medicinal herbs, has been extensively used for thousands of years in the treatment of infectious diarrhea, heat-clearing and detoxification in Traditional Chinese Medicine $(8,9)$. Studies have demonstrated that BBR possesses different pharmacological activities involving multiple biological mechanisms, and may be utilized for the treatment of various diseases, including diabetes, metabolic syndrome and various types of cancer (9-14). It was reported that BBR has a beneficial effect on patients with diabetes and obesity, partly by stimulating adenosine monophosphate kinase activity (15). Other studies have demonstrated that BBR sensitized ovarian cancer cells to cisplatin through the microRNA-93/phosphatase and tensin homologue/Akt signaling axis $(13,16,17)$.

However, whether BBR exerts a suppressive effect on LPS-induced adenomyosis via inhibiting TLR4-mediated stromal cell invasion has remained elusive. The aim of the present study was to investigate the effect of BBR on LPS-induced ectopic endometrial stromal cells (EESCs) and the development of adenomyosis. 


\section{Materials and methods}

Reagents. BBR and LPS were purchased from Sigma-Aldrich (Merck KGaA, Darmstadt, Germany). ELISA kits for interleukin (IL)-6 (cat. no. HM10205), IL-8 (cat. no. HM10222) and transforming growth factor (TGF)- $\beta$ (cat. no. HM10058) were purchased from Bio-Swamp (Shanghai, China). Antibodies against epithelial growth factor (EGF) (cat. no. Ab9695) and matrix metalloproteinase (MMP)2 (cat. no. Ab92536) were purchased from Abcam (Cambridge, MA, USA). Antibodies against vascular endothelial growth factor (VEGF) (cat. no. AF5131) were purchased from Affinity Biosciences (Cincinnati, OH, USA), while antibody against GAPDH (cat. no. 5174) was purchased from Cell Signaling Technologies (Danvers, MA, USA). The secondary antibodies donkey anti-goat horseradish peroxidase (HRP) (cat. no. A0181), goat anti-rabbit HRP (cat. no. A0208) and goat anti-mouse HRP (cat. no. A0216) were purchased from Beyotime Institute of Technology (Haimen, China).

Subjects and specimens. Between August 2016 to October 2016, a total of three patients with adenomyosis between 22 and 40 years of age undergoing laparoscopy for pelvic pain or/and dysmenorrhea at Yangpu Hospital affiliated to Tongji University School of Medicine (Shanghai, China) were recruited. All of them had regular menstrual cycles (28-32 days) and had not been on any hormonal medication 3 months prior to the initiation of the study. The phase of the menstrual cycle was the early proliferative phase. The diagnosis was confirmed by pathology, which excluded the presence of any other gynecological diseases. Adenomyosis was diagnostically confirmed by the macroscopic appearance of the biopsy samples according to the published criteria (18) and the final diagnosis was mainly based on histological examination. All fresh tissue specimens were collected in PBS with added $100 \mathrm{U} / \mathrm{ml}$ penicillin, $100 \mu \mathrm{g} / \mathrm{ml}$ streptomycin, stored at $4^{\circ} \mathrm{C}$, and processed within 1-6 $\mathrm{h}$. The study was approved by the Research Ethics Committee of Tongji University (Shanghai, China) and written informed consent was obtained from all patients.

Cell collection and culture. EESCs (EESC1, EESC2 and EESC3, respectively) were isolated and characterized as described previously (19-21). In brief, the tissues were washed twice with sterile PBS under aseptic conditions, minced into small pieces, digested by incubation with Dulbecco's modified Eagle's medium (DMEM)/F12 (Hyclone; GE Healthcare Life Sciences, South Logan, UT, USA) supplemented with $1 \mathrm{mg} / \mathrm{ml}$ collagenase (Serva Electrophoresis GmbH, Heidelberg, Germany) and 10\% fetal bovine serum (FBS; Gibco; Thermo Fisher Scientific, Inc., Waltham, MA, USA) for $2 \mathrm{~h}$ at $37^{\circ} \mathrm{C}$ and subsequently filtrated with a $70-\mathrm{mm}$ nylon cell strainer. The resultant supernatant was centrifuged at $111 \mathrm{x} \mathrm{g}$ for $5 \mathrm{~min}$ at room temperature, and the pellets were re-suspended and cultured with DMEM/F12 containing $10 \%$ FBS, $100 \mathrm{IU} / \mathrm{ml}$ penicillin and $100 \mathrm{mg} / \mathrm{ml}$ streptomycin at $37^{\circ} \mathrm{C}$ in a humidified air atmosphere containing $5 \% \mathrm{CO}_{2}$. Identification of the isolated cells was performed with anti-vimentin and mouse anti-CK19 antibodies by immunohistochemistry (22).
Cell proliferation assay. Cells (5.0x10 $10^{3} /$ well) were seeded in 96-well plates and stimulated with different concentrations (50, 100 and $200 \mu \mathrm{M})$ of BBR or/and LPS $(100 \mathrm{ng} / \mathrm{ml})$ for 24,48 or $72 \mathrm{~h}$. Cell proliferation was detected by using the Cell Count Kit-8 (CCK-8; Signalway Antibody, LLC, College Park, MD, USA) according to the manufacturer's protocol. The optical density (OD) was detected at a wavelength of $450 \mathrm{~nm}$ with a microplate reader (Bio-Rad Laboratories, Inc., Hercules, CA, USA). The relative cell viability was calculated using following formula: (OD of sample/OD of blank control) $\mathrm{x} 100 \%$. Each assay was performed in triplicate.

Cell cycle analysis. The cell cycle was evaluated by propidium iodide (PI; 7 Sea Biotech, Shanghai, China) staining and analysis using a flow cytometer (BD Biosciences). Cells were seeded on 6 -well plates at $3 \times 10^{4}$ cells/well, treated with BBR $(200 \mu \mathrm{M})$ or/and LPS $(100 \mathrm{ng} / \mathrm{ml})$ for $48 \mathrm{~h}$, washed in PBS and re-suspended in staining solution containing $20 \mu \mathrm{g} / \mathrm{ml} \mathrm{PI}$ and $100 \mu \mathrm{g} / \mathrm{ml}$ RNase A (Beijing Solarbio Science \& Technology Co., Ltd., Beijing, China). Experiments were performed in triplicate. G1, S and G2/M fractions were quantified with FlowJo 7.6.1 software (FlowJo, LLC, Ashland, OR, USA). The experiments were performed in triplicate.

Annexin V-fluorescein isothiocyanate (FITC)/PI apoptosis assay. Apoptotic cells were analyzed using an Annexin V-FITC/PI double staining procedure. Cells were treated with BBR $(200 \mu \mathrm{M})$ and/or LPS $(100 \mathrm{ng} / \mathrm{ml})$ for $48 \mathrm{~h}$. Subsequently, cells were digested into single cell suspensions using EDTA-free trypsin (Beijing Solarbio Science $\&$ Technology Co., Ltd.) and then stained according to the instructions provided with the Annexin V-FITC/PI Apoptosis Detection kit (Beyotime Institute of Biotechnology). The stained cells were analyzed within 10-15 min by flow cytometry (BD Biosciences). At least $2 \times 10^{4}$ cells were acquired for each sample. The experiments were performed in triplicate.

Reverse-transcription quantitative polymerase chain reaction $(R T-q P C R)$. PCR was performed using an ABI 7300 instrument (Applied Biosystems; Thermo Fisher Scientific, Inc.) and SYBR-Green Master Mix (cat. no. K0223; Thermo Fisher Scientific, Inc.). The primers had the following sequences: IL-6 forward, 5'-AGCCACTCACCTCTTCAGAAC-3' and reverse, 5'-GCCTCTTTGCTGCTTTCACAC-3'; IL-8 forward, 5'-CAAGAGCCAGGAAGAAAC-3' and reverse, 5'-TGGTCCACTCTCAATCAC-3'; TGF- $\beta 1$ forward, 5 '-GAC TACTACGCCAAGGAGGTC-3' and reverse, 5'-GAGAGC AACACGGGTTCAG-3'; VEGF forward, 5'-ATTTCTGGG ATTCCTGTAG-3' and reverse, 5'-CAGTGAAGACACCAA TAAC-3'; EGF forward, 5'-GAAACTGTTGGGAGAGGA ATCG-3' and reverse, 5'-AGAGCAAGGCAAAGGCTT AG-3'; MMP2 forward, 5'-TTGACGGTAAGGACGGAC TC-3' and reverse, 5'-GGCGTTCCCATACTTCACAC-3'; GAPDH forward, 5'-CACCCACTCCTCCACCTTTG-3' and reverse, 5'-CCACCACCCTGTTGCTGTAG-3'. GAPDH was used as an internal control. EESCs $\left(5.0 \times 10^{5} /\right.$ well) were seeded in 6-well plates and treated with BBR $(200 \mu \mathrm{M})$ for $48 \mathrm{~h}$. Total RNA was extracted using TRIzol reagent (Invitrogen; Thermo Fisher Scientific, Inc.) according to the manufacturer's instructions. Complementary DNA was synthesized by using 
RT Reagent kit (cat. no. K1622; Fermentas, Thermo Fisher Scientific, Inc.) at $37^{\circ} \mathrm{C}$ for $1 \mathrm{~h}$ and $85^{\circ} \mathrm{C}$ for $5 \mathrm{~min}$. The PCR cycling conditions were as follows: $95^{\circ} \mathrm{C}$ for $10 \mathrm{~min}$, followed by 40 cycles of $15 \mathrm{sec}$ at $95^{\circ} \mathrm{C}$ and $60^{\circ} \mathrm{C}$ for $1 \mathrm{~min}$. Verification of specific product amplification was performed by dissociation curve analysis. The gene expression was calculated using the $2^{-\Delta \Delta \mathrm{Cq}}$ method (23). All results are expressed as the mean of three replicates.

Western blot analysis. Cell lysates were prepared with radioimmunoprecipitation assay buffer. The protein concentration was measured by a bicinchoninic acid assay (Thermo Fisher Scientific, Inc.). The supernatant with an equal amount of protein (15 $\mu \mathrm{g}$ protein/lane) was separated by $10 \%$ SDS-PAGE. Proteins then were blotted onto nitrocellulose membranes (EMD Millipore, Billerica, MA, USA) and incubated with primary antibodies for $2 \mathrm{~h}$ at room temperature, followed by the corresponding secondary antibodies for $1 \mathrm{~h}$ at room temperature. The bound antibodies were visualized by enhanced chemiluminescence and quantified by densitometry using ChemiDocTM XRS+ image analyzer (Bio-Rad Laboratories, Inc., Hercules, CA, USA). Densitometric analyses of the bands were adjusted with GAPDH. Each assay was performed in triplicate.

ELISA. The secretion of IL-6, IL- 8 and TGF- $\beta$ in culture medium was respectively determined using the human IL-6, IL- 8 and TGF- $\beta$ ELISA kits (Bio-Swamp), according to the protocol provided by the manufacturer. The EESCs were treated with LPS or/and BBR. The culture medium was collected and centrifuged for $1 \mathrm{~min}$ at $111 \mathrm{x} \mathrm{g}$ at $4^{\circ} \mathrm{C}$, and the secreted IL- 6 , IL- 8 and TGF- $\beta$ in the culture medium were measured spectrophotometrically in an ELISA reader at $450 \mathrm{~nm}$. The absolute concentration of IL- 6 , IL- 8 and TGF- $\beta$ in the culture medium was calculated from a standard curve.

Statistical analysis. Data analysis was performed using SPSS 20.0 software (IBM Corp., Armonk, NY, USA). Values are expressed as the mean \pm standard error of the mean. Comparisons between multiple groups were made by analysis of variance followed by Tukey's honest post hoc test, or by the Wilcoxon test. $\mathrm{P}<0.05$ was considered to indicate a statistically significant difference.

\section{Results}

BBR inhibits the proliferation of LPS-induced EESCs. To evaluate the effects of BBR on the proliferation of LPS-induced EESCs, the amount of viable cells was detected by a CCK-8 assay. In accordance with the results of a previous study by our group, EESCs presented with a maximum increase in the levels of cell growth at $48 \mathrm{~h}$ and in response to $10-100 \mathrm{ng} / \mathrm{ml}$ LPS (7), and thus, $100 \mathrm{ng} / \mathrm{ml}$ was selected as the optimal concentration of LPS in the subsequent experiments. As presented in Fig. 1, EESCs were treated with $100 \mathrm{ng} / \mathrm{ml}$ LPS with or without different concentrations of BBR for 24,48 and $72 \mathrm{~h}$. The results revealed that LPS significantly increased the proliferation and viability of EESCs compared with that in the blank group $(\mathrm{P}<0.05)$. Combined treatment with BBR markedly decreased the LPS-induced cell proliferation in a time- and dose-dependent

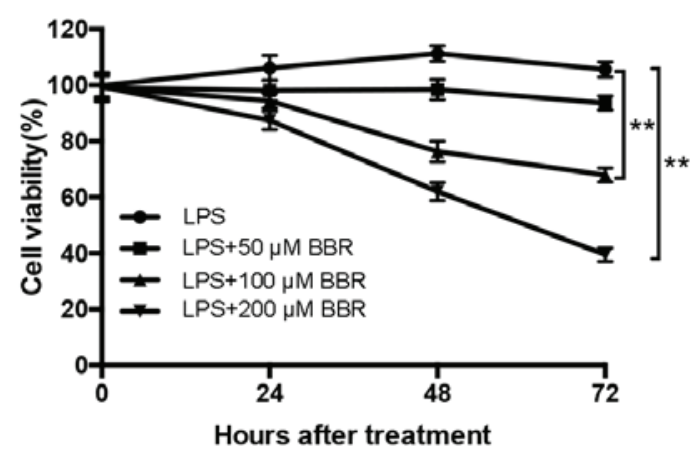

Figure 1. BBR inhibits the proliferation of LPS-induced EESCs. EESCs were exposed to LPS or LPS plus various concentrations of BBR for the indicated periods. Cell viability was measured by a Cell Counting Kit- 8 assay. Data were obtained from at least three independent experiments. ${ }^{* *} \mathrm{P}<0.01$. BBR, berberine; LPS, lipopolysaccharide; EESCs, ectopic endometrial stromal cells.

manner. The amount of viable EESCs after treatment with 100 or $200 \mu \mathrm{M}$ BBR combined with LPS was significantly lower than that after treatment with $50 \mu \mathrm{M}$ BBR combined with LPS. Compared with 24-h BBR+LPS treatment, 48- and 72-h BBR+LPS treatment had a significantly higher inhibitory effect on the growth of EESCs. Therefore, the concentration of $200 \mu \mathrm{M}$ was selected as the optimal BBR concentration combined with LPS in the subsequent experiments.

BBR induces cell cycle arrest of LPS-induced EESCs. To determine whether BBR affected the cell cycle of LPS-induced EESCs, the cell cycle distribution was evaluated by flow cytometry using PI staining. As presented in Fig. 2, LPS treatment significantly decreased the number of EESCs in G0/G1 phase and increased the number of EESCs in G2/M phase as compared with that in the control group. The combination of BBR and LPS could markedly induced cell cycle arrest of EESCs in G0/G1 phase, which may have been the cause of BBR-induced inhibition of cell proliferation.

BBR increases apoptosis of LPS-induced EESCs. To explore the apoptotic effect of BBR on LPS-induced EESCs, apoptosis was evaluated by using an Annexin V-FITC/PI staining assay. As presented in Fig. 3, compared with the control cells, treatment of EESCs with LPS for $48 \mathrm{~h}$ led to a decreased early (lower right quadrant) and late (upper right quadrant) apoptotic rate. The combination of BBR and LPS significantly increased the number of apoptotic EESCs as compared with that in the LPS-induced group. These results suggested that BBR has a marked apoptosis-promoting effect on LPS-induced EESCs.

BBR inhibits the expression of IL-6, IL-8, TGF- $\beta, E G F, V E G F$ and MMP 2 in LPS-induced EESCs. To evaluate whether BBR affected the development of LPS-induced EESCs, the expression of relevant markers, which are crucial for the infiltration and proliferation of EESCs, were detected by RT-qPCR, ELISA and western blot analysis. The results demonstrated that LPS significantly increased the mRNA (Fig. 4A) and protein (Fig. 4B-D) expression of IL-6, IL-8, TGF- $\beta$, EGF, VEGF and MMP2 as compared with that in the control group. After treatment of EESCs with BBR and LPS, the mRNA (Fig. 4A) and protein (Fig. 4B-D) levels of IL-6, IL-8, TGF- $\beta$, EGF, VEGF 


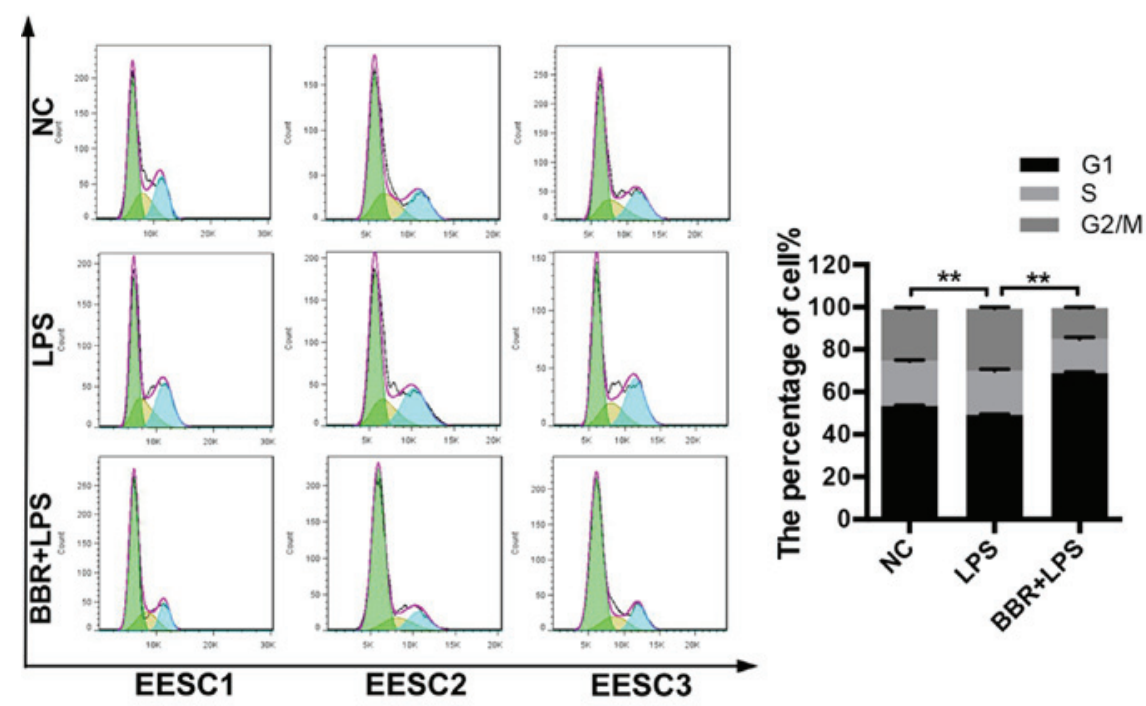

Figure 2. BBR induces cell cycle arrest of LPS-induced EESCs. EESCs from 3 different patients were treated with LPS or LPS+BBR for $48 \mathrm{~h}$. The cell cycle was evaluated by flow cytometry using propidium iodide staining. The green, yellow and blue sections respectively referred to G0/G1, S and G2/M phase. Data were obtained from at least three independent experiments. ${ }^{* *} \mathrm{P}<0.01$. BBR, berberine; LPS, lipopolysaccharids; EESCs, ectopic endometrial stromal cells; $\mathrm{NC}$, negative control.

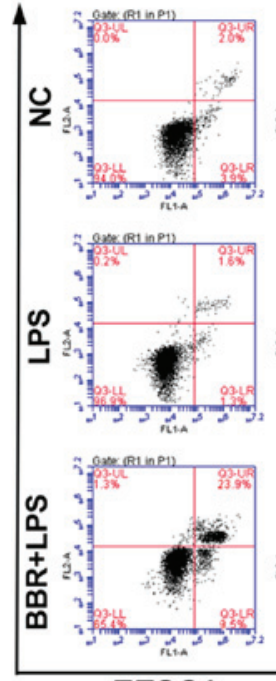

EESC1
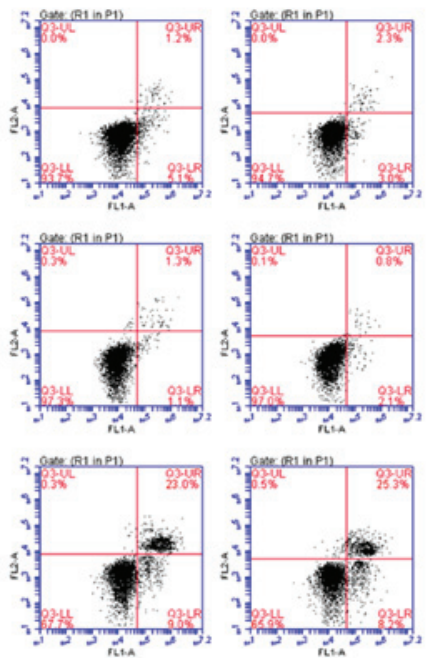

EESC2

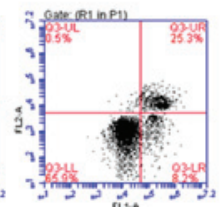

EESC3

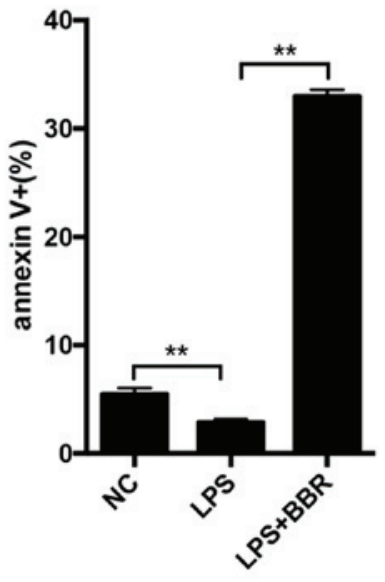

Figure 3. BBR increases apoptosis of LPS-induced EESCs. EESCs from 3 different patients were treated with LPS or LPS+BBR for 48 h. The number of cells undergoing apoptosis was detected by flow cytometry using Annexin V-fluorescein isothiocyanate/propidium iodide double staining. Data were obtained from at least three independent experiments. ${ }^{* *} \mathrm{P}<0.01$. BBR, berberine; LPS, lipopolysaccharide; EESCs, ectopic endometrial stromal cells; NC, negative control; Q3-UR, upper right; Q3-LR, lower right; Q3-LL, lower left; Q3-UL, upper left.

and MMP2 were significantly decreased as compared with those in the LPS-treated group. These results indicated that BBR may inhibit the LPS-induced invasion and proliferation of EESCs, which is associated with adenomyosis.

\section{Discussion}

LPS-mediated TLR4 signaling as a first line of defense against microbial infection has been extensively studied in the past decades. The improper inflammatory response induced by LPS/TLR4 signaling has the potential to cause inflammatory disorders $(24,25)$. Various studies have indicated that local and chronic inflammatory reactions in the uterus and peritoneal environment are likely to be associated with the pathogenesis of endometriosis/adenomyosis $(7,26,27)$. Azuma et al (26) reported that LPS promoted the development of endometriosis through the $N F-\kappa B$ pathway in a murine model. A previous study by our group demonstrated that LPS/TLR4-mediated stromal cells acquire an invasive phenotype and are implicated in the pathogenesis of adenomyosis, which shed light on the mechanism underlying adenomyosis and provided a novel potential strategy for the clinical treatment of the disease (7).

$\mathrm{BBR}$ as an isoquinoline derivative alkaloid has been demonstrated to have diverse pharmacological activities involving multiple biological mechanisms, which may be 
A

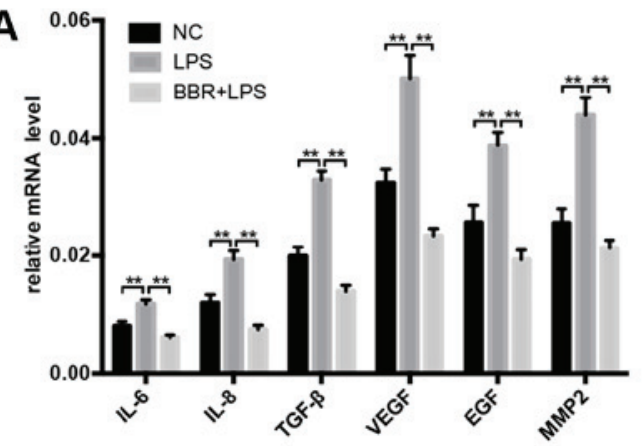

B

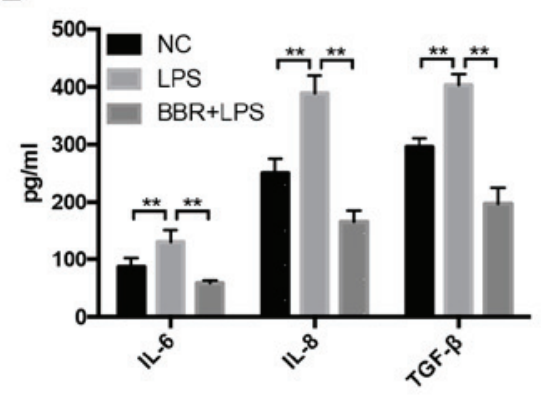

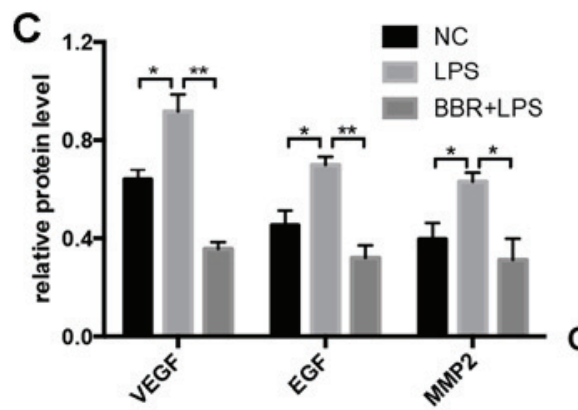

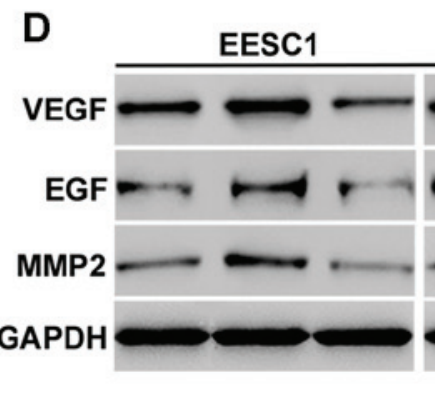

EESC2

EESC3

Figure 4. BBR inhibits the expression of IL-6, IL-8, TGF- $\beta$, EGF, VEGF and MMP2 in LPS-induced EESCs. EESCs from 3 different patients were treated with LPS or LPS+BBR for $48 \mathrm{~h}$. The expression levels of IL-6, IL-8 and TGF- $\beta$ in EESCs were detected by (A) RT-qPCR and (B) ELISA. The expression levels of EGF, VEGF and MMP2 in EESCs was detected by (A) RT-qPCR and (C and D) western blot analysis. Data were obtained from at least three independent experiments. " $\mathrm{P}<0.05,{ }^{* *} \mathrm{P}<0.01$. BBR, berberine; LPS, lipopolysaccharide; EESCs, ectopic endometrial stromal cells; NC, negative control; IL, interleukin; TGF, transforming growth factor; EGF, epithelial growth factor; VEGF, vascular endothelial growth factor; MMP, matrix metalloproteinase; RT-qPCR, reverse transcription-quantitative polymerase chain reaction.

utilized for the treatment of a wide variety of diseases (9-14). Previous studies have demonstrated that BBR acts as an LPS antagonist and blocks LPS/TLR4 signaling in an LPS-induced animal model (28-30). Li et al (30) reported that BBR protects against LPS-induced intestinal injury in mice via reducing enterocyte apoptosis, inhibiting the TLR4 pathway and decreasing neutrophil infiltration. Huang et al (29) reported that BBR exerted a potent anti-inflammatory effect by suppressing the LPS-evoked secretion of the proinflammatory cytokines IL-1 $\beta$, IL- 6 and TNF $\alpha$ and attenuated the activation of LPS-TLR4-cytokines/NF- $\mathrm{B}$ signaling pathways in the mouse macrophage-like cell line RAW264.7. However, the effect of BBR on LPS-induced adenomyosis has remained elusive. In the present study, a CCK- 8 assay and flow cytometric analysis demonstrated that BBR significantly and time- and dose-dependently inhibited the proliferation and significantly inhibited the cell cycle progression of LPS-induced EESCs.

Adenomyosis, as a specific type of endometriosis, features certain alterations in apoptosis, invasion and angiogenesis compared with healthy controls. Indeed, numerous cytokines have been demonstrated to have a major role in the pathogenesis of adenomyosis $(7,21,31,32)$. The present study focused on IL-6, IL-8, TGF- $\beta$, EGF, VEGF and MMP2 to investigate whether BBR inhibited the invasion, inflammatory response and maintenance of LPS-induced stromal cells from adenomyosis tissues. The results revealed that BBR significantly inhibited the mRNA and protein expression of IL-6, IL-8, TGF- $\beta$, EGF, VEGF and MMP2 in LPS-induced EESCs. Various studies confirmed that various cytokines, including IL-6, IL-8, IL-10, TNF- $\alpha$ and TGF- $\beta$, synthesized and secreted by peritoneal macrophages may contribute to the adhesion, invasion and proliferation of endometrial cells and the progression of endometriosis (33-36). Yang et al (32) reported that abnormal secretion of IL-6 and IL- 8 from endometrial stromal cells may have a role in the formation of ectopic islands in adenomyosis. Furthermore, the role of EGF, VEGF and TGF- $\beta$ implicated in inflammation, angiogenesis and the pathophysiology of endometriosis has been well established (36-38). Cell invasion is a vital hallmark of tumor formation and progression. Indeed, upregulation of several MMPs has also been linked to the pathogenesis of adenomyosis $(39,40)$. Weigel et al (41) demonstrated obvious differences in the expression pattern of MMP2 and MMP9 in different stages of endometriosis and suggested that these markers may be used to evaluate the aggressiveness and invasiveness of endometriotic lesions.

In conclusion, the present study indicated that BBR has the ability to induce apoptosis and inhibit the proliferation and invasive phenotypes of LPS-induced stromal cells of adenomyosis tissues. The present findings shed light on the potential mechanism of BBR targeting the adenomyosis cells stimulated by LPS and provided a novel therapeutic strategy for the clinical treatment of the disease. However, further investigation of the molecular mechanism in vivo and randomized controlled trials are required for the clinical application of BBR.

\section{Acknowledgements}

This study was supported by grants from the Shanghai Municipal Commission of Health and Family Planning (grant no. ZA2015A33). 


\section{References}

1. Harvey J and Warwick I: Endometriosis. BMJ 340: c2661, 2010.

2. Korzekwa A, Łupicka M, Socha B, Mannelli Ch and Skarzynski DJ: Is adenomyosis a problem in reproduction and fertility? Pol J Vet Sci 17: 187-194, 2014.

3. Farquhar C and Brosens I: Medical and surgical management of adenomyosis. Best Pract Res Clin Obstet Gynaecol 20: 603-616, 2006.

4. Lousse JC, Van Langendonckt A, Defrere S, Ramos RG, Colette S and Donnez J: Peritoneal endometriosis is an inflammatory disease. Front Biosci (Elite Ed) 4: 23-40, 2012.

5. Sheldon IM and Bromfield JJ: Innate immunity in the human endometrium and ovary. Am J Reprod Immunol 66 (Suppl 1): S63-S71, 2011.

6. Bertschi D, McKinnon BD, Evers J, Bersinger NA and Mueller MD: Enhanced inflammatory activity of endometriotic lesions from the rectovaginal septum. Mediators Inflamm 2013 $450950,2013$.

7. Guo J, Chen L, Luo N, Li C, Chen R, Qu X, Liu M, Kang L and Cheng Z: LPS/TLR4-mediated stromal cells acquire an invasive phenotype and are implicated in the pathogenesis of adenomyosis. Sci Rep 6: 21416, 2016.

8. Pan L, Wang W, Shi F, Zhou J, Zhang M, Zhu H and Zeng M: Exploratory pharmacokinetics of Geniposide in rat model of cerebral ischemia orally administered with or without Baicalin and/or Berberine. Evid Based Complement Alternat Med 2013: $349531,2013$.

9. Sun Y, Xun K, Wang Y and Chen X: A systematic review of the anticancer properties of berberine, a natural product from Chinese herbs. Anticancer Drugs 20: 757-769, 2009.

10. Kong WJ, Zhang H, Song DQ, Xue R, Zhao W, Wei J, Wang YM, Shan N, Zhou ZX, Yang P, et al: Berberine reduces insulin resistance through protein kinase $\mathrm{C}$-dependent up-regulation of insulin receptor expression. Metabolism 58: 109-119, 2009.

11. Singh IP and Mahajan S: Berberine and its derivatives: A patent review (2009-2012). Expert Opin Ther Pat 23: 215-231, 2013

12. Yin J, Zhang $\mathrm{H}$ and Ye J: Traditional chinese medicine in treatment of metabolic syndrome. Endoc Metab Immune Disord Drug Targets 8: 99-111, 2008.

13. Jin $\mathrm{P}$, Zhang $\mathrm{C}$ and $\mathrm{Li} \mathrm{N}$ : Berberine exhibits antitumor effects in human ovarian cancer cells. Anticancer Agents Med Chem 15: 511-516, 2015.

14. Li J, Cao B, Liu X, Fu X, Xiong Z, Chen L, Sartor O, Dong Y and Zhang H: Berberine suppresses androgen receptor signaling in prostate cancer. Mol Cancer Ther 10: 1346-1356, 2011.

15. Lee YS, Kim WS, Kim KH, Yoon MJ, Cho HJ, Shen Y, Ye JM, Lee $\mathrm{CH}$, Oh WK, Kim CT, et al: Berberine, a natural plant product, activates AMP-activated protein kinase with beneficial metabolic effects in diabetic and insulin-resistant states. Diabetes 55: 2256-2264, 2006.

16. Chen Q, Qin R, Fang Y and Li H: Berberine sensitizes human ovarian cancer cells to cisplatin through miR-93/PTEN/Akt signaling pathway. Cell Physiol Biochem 36: 956-965, 2015.

17. Marverti G, Ligabue A, Lombardi P, Ferrari S, Monti MG Frassineti C and Costi MP: Modulation of the expression of folate cycle enzymes and polyamine metabolism by berberine in cisplatin-sensitive and -resistant human ovarian cancer cells. Int J Oncol 43: 1269-1280, 2013.

18. Exacoustos C, Manganaro L and Zupi E: Imaging for the evaluation of endometriosis and adenomyosis. Best Pract Res Clin Obst Gynaecol 28: 655-681, 2014.

19. Rajaei S, Mirahmadian M, Jeddi-Tehrani M, Tavakoli M, Zonoobi M, Dabbagh A and Zarnani AH: Effect of 1,25(OH)2 vitamin D3 on cytokine production by endometrial cells of women with repeated implantation failure. Gynecol Endocrinol 28: 906-911, 2012

20. Wan L, Zou Y, Wan LH, Wang LQ, Huang MZ, Wu J, Zhu YB and Huang OP: Tanshinone IIA inhibits the proliferation, migration and invasion of ectopic endometrial stromal cells of adenomyosis via 14-3-3zeta downregulation. Arch Gynecol Obstet 292: 1301-1309, 2015.

21. Yang JH, Wu MY, Chen CD, Chen MJ, Yang YS and Ho HN: Altered apoptosis and proliferation in endometrial stromal cells of women with adenomyosis. Hum Reprod 22: 945-952, 2007.
22. Bruse C, Guan Y, Carlberg M, Carlström K and Bergqvist A: Basal release of urokinase plasminogen activator, plasminogen activator inhibitor-1, and soluble plasminogen activator receptor from separated and cultured endometriotic and endometrial stromal and epithelial cells. Fertil Steril 83 (Suppl 1): S1155-S1160, 2005.

23. Livak KJ and Schmittgen TD: Analysis of relative gene expression data using real-time quantitative PCR and the 2(-Delta Delta C(T)) method. Methods 25: 402-408, 2001.

24. Miller SI, Ernst RK and Bader MW: LPS, TLR4 and infectious disease diversity. Nat Rev Microbiol 3: 36-46, 2005.

25. Lu YC, Yeh WC and Ohashi PS: LPS/TLR4 signal transduction pathway. Cytokine 42: 145-151, 2008.

26. Azuma Y, Taniguchi F, Nakamura K, Nagira K, Khine YM, Kiyama T1,Uegaki T, Izawa M and Harada T: Lipopolysaccharide promotes the development of murine endometriosis-like lesions via the nuclear factor-kappa B pathway. Am J Reprode Immunol 77, 2017.

27. Allhorn S, Böing C, Koch AA, Kimmig R and Gashaw I: TLR3 and TLR4 expression in healthy and diseased human endometrium. Reprod Biol Endocrinol 6: 40, 2008.

28. Chu M, Ding R, Chu ZY, Zhang MB, Liu XY, Xie SH, Zhai YJ and Wang YD: Role of berberine in anti-bacterial as a high-affinity LPS antagonist binding to TLR4/MD-2 receptor. BMC Complement Alternat Med 14: 89, 2014.

29. Huang LH, Pan XP, Gong KR and Shao G: Anti-inflammatory effects of three kinds of traditional Mongolian medicine monomer and its combination on LPS-stimulated RAW264.7 macrophages. Eur Rev Med Pharmacol Sci 20: 950-958, 2016.

30. Li HM, Wang YY, Wang HD, Cao WJ, Yu XH, Lu DX, Qi RB, Hu CF and Yan YX: Berberine protects against lipopolysaccharide-induced intestinal injury in mice via alpha 2 adrenoceptor-independent mechanisms. Acta Pharmacol Sin 32: 1364-1372, 2011

31. Benagiano G, Brosens I and Habiba M: Structural and molecular features of the endomyometrium in endometriosis and adenomyosis. Hum Reprod Update 20: 386-402, 2014.

32. Yang JH, Chen MJ, Wu MY, Chen YC, Yang YS and Ho HN: Decreased suppression of interleukin-6 after treatment with medroxyprogesterone acetate and danazol in endometrial stromal cells of women with adenomyosis. Fertil Steril 86: 1459-1465, 2006.

33. May KE, Conduit-Hulbert SA, Villar J, Kirtley S, Kennedy SH and Becker CM: Peripheral biomarkers of endometriosis: A systematic review. Hum Reprod Update 16: 651-674, 2010.

34. Younis A, Hawkins K, Mahini H, Butler W and Garelnabi M: Serum tumor necrosis factor- $\alpha$, interleukin- 6 , monocyte chemotactic protein-1 and paraoxonase-1 profiles in women with endometriosis, PCOS, or unexplained infertility. J Assist Reprod Genet 31: 1445-1451, 2014.

35. Khan KN, Masuzaki H, Fujishita A, Kitajima M, Hiraki K, Sekine I, Matsuyama T and Ishimaru T: Interleukin-6- and tumour necrosis factor alpha-mediated expression of hepatocyte growth factor by stromal cells and its involvement in the growth of endometriosis. Hum Reprod 20: 2715-2723, 2005.

36. Sotnikova N, Antsiferova I and Malyshkina A: Cytokine network of eutopic and ectopic endometrium in women with adenomyosis. Am J Reprod Immunol 47: 251-255, 2002.

37. Omwandho CO, Konrad L, Halis G, Oehmke F and Tinneberg HR: Role of TGF-betas in normal human endometrium and endometriosis. Hum Reprod 25: 101-109, 2010.

38. Kang S, Zhao J, Liu Q, Zhou R, Wang N and Li Y: Vascular endothelial growth factor gene polymorphisms are associated with the risk of developing adenomyosis. Environ Mol Mutagen 50: 361-366, 2009.

39. Yang JH, Wu MY, Chen MJ, Chen SU, Yang YS and Ho HN: Increased matrix metalloproteinase-2 and tissue inhibitor of metalloproteinase-1 secretion but unaffected invasiveness of endometrial stromal cells in adenomyosis. Fertil Steril 91 (5 Suppl): 2193-2198, 2009.

40. Yi KW, Kim SH, Ihm HJ, Oh YS, Chae HD, Kim CH and Kang BM: Increased expression of p21-activated kinase 4 in adenomyosis and its regulation of matrix metalloproteinase-2 and -9 in endometrial cells. Fertil Steril 103: 1089-1097.e2, 2015.

41. Weigel MT, Krämer J, Schem C, Wenners A, Alkatout I, Jonat W, Maass N and Mundhenke C: Differential expression of MMP-2, MMP-9 and PCNA in endometriosis and endometrial carcinoma. Eur J Obstet Gynecol Reprod Biol 160: 74-78, 2012. 